\title{
Rheology of Cohesive Granular Media: Shear Banding, Hysteresis, and Nonlocal Effects
}

\author{
Sandip Mandal@, Maxime Nicolas®, and Olivier Pouliquen* \\ Aix Marseille Univ, CNRS, IUSTI, 13453 Marseille, France
}

(Received 3 November 2020; revised 25 February 2021; accepted 15 March 2021; published 20 April 2021)

\begin{abstract}
Powders or cohesive granular materials are widely handled in industries. However, our understanding of the rheology of these materials is limited. Here, we provide a comprehensive analysis of the rheology of a cohesive granular medium, sheared in a normal-stress-imposed plane shear cell over a wide range of shear rate, employing numerical simulations. At high imposed shear rates, the flow is homogeneous, and the rheology is well described by the existing scaling laws, involving the inertial number and the "effective" cohesion number [S. Mandalet al., Insights into the Rheology of Cohesive Granular Media, Proc. Natl. Acad. Sci. U.S.A. 117, 8366 (2020)]. However, at low imposed shear rates, the flow is inhomogeneous, exhibiting the coexistence of flowing and nonflowing regions in the material, popularly known as shear banding. We thoroughly analyze the crucial features of this shear-banded flow regime and discuss striking similarities between the shear banding for granular media and other complex fluids. We reveal that the occurrence of shear banding is related to the existence of a nonmonotonic intrinsic rheological curve and that increasing adhesion increases the nonmonotonicity and the tendency toward shear localization. A simple theoretical model based on a nonlocal rheological model coupled with a nonmonotonic flow curve is proposed and is shown to successfully reproduce all the key features of the shear banding observed in the numerical simulations. The results have important implications for the handling of powders in industries.
\end{abstract}

DOI: 10.1103/PhysRevX.11.021017

Subject Areas: Fluid Dynamics, Soft Matter

\section{INTRODUCTION}

Many industrial processes handle powders or cohesive granular media and are confronted with flow and jamming problems in production lines. The notion of "flowability" based on standardized tests [1-3] has been introduced to characterize the ability of powders to flow and to help improve designing of industrial processes. However, the physical basis of this concept remains unclear. Hence, a detailed understanding of the flow properties and rheology of cohesive granular media would be useful. In this paper, we fill this gap by performing intensive numerical simulations of the flow of a cohesive granular medium in a plane shear configuration.

In the absence of adhesion between the grains, the rheology of rigid granular media is well described, at first order, by the simple $\mu(I)$ rheology [4-6]. When an assembly of rigid grains of (mean) diameter $d$ and density $\rho_{p}$ is sheared at a shear rate $\dot{\gamma}$ under a confining normal stress $\sigma_{z z}$, the resulting shear stress and volume fraction are

\footnotetext{
*olivier.pouliquen@univ-amu.fr
}

Published by the American Physical Society under the terms of the Creative Commons Attribution 4.0 International license. Further distribution of this work must maintain attribution to the author(s) and the published article's title, journal citation, and DOI. controlled by a single dimensionless number called the inertial number $I=\dot{\gamma} d / \sqrt{\sigma_{z z} / \rho_{p}}$, and the constitutive laws are reduced to a friction coefficient $\mu(I)$ and a volume fraction $\phi(I)$, which are functions of $I$. To understand how this picture is modified in the presence of adhesion between the grains, several numerical studies [7-12] have simulated the flow of cohesive grains using different models for the adhesive forces (capillary attraction, van der Walls force, etc.) between the grains. A key parameter of these models is the adhesion force $N_{c}$, defined as the force necessary to detach two particles in contact. The introduction of this new force scale $N_{c}$ introduces a new dimensionless number called the cohesion number $C=N_{c} /\left(\sigma_{z z} d^{2}\right)$ [7-12], which compares the detachment force to the typical compression force due to the confining normal stress. The friction coefficient and the volume fraction are then assumed to be functions of both $I$ and $C$, and it has been shown that $\mu(I, C)$ and $\phi(I, C)$ increase and decrease, respectively, with increasing $I$ and $C$ [7-11]. Scaling laws have been proposed, allowing for a simpler description of these functions in terms of a modified inertial number [12].

In our previous study [13] involving the flow down a rough inclined plane, we have shown that this description of the rheology of cohesive granular media is incomplete. While the cohesion number $C$ was shown to control the cohesive properties of the material at incipient failure, it failed to describe the rheology in the dynamic regime 
completely, as the material properties of the grains like the stiffness $k_{n}$ and the inelasticity, quantified through the quality factor $Q$, also came into the picture by affecting the flow. For the same interparticle adhesion $N_{c}$, stiffer (high $k_{n}$ ) and less dissipative (high $Q$ ) grains yielded a less cohesive flow. We showed that an "effective" adhesion $N_{c}^{\text {eff }}$ could be defined to collate the effects of $N_{c}, k_{n}$, and $Q$ and proposed modified constitutive relations as $\mu=\mu\left(I, C^{\text {eff }}\right)$ and $\phi=\phi\left(I, C^{\text {eff }}\right)$, where $C^{\text {eff }}=N_{c}^{\text {eff }} /\left(\sigma_{z z} d^{2}\right)$ is the effective cohesion number. However, using the inclined plane geometry, we were unable to show the bridging between the static rheological branch controlled by $C$ and the dynamic branch controlled by $I$ and $C^{\text {eff }}$. Here, using the plane shear cell, we probe the dynamic branch at low inertial numbers, where we discover a rich dynamics with a systematic development of shear bands.

Shear banding (SB) is a phenomenon which leads to the coexistence of regions of different shear rates in a material, sheared under a homogeneous shear stress distribution. It is commonly observed in a broad class of complex fluids like emulsions, colloidal suspensions, polymer solutions, wormlike micelles, or foams [14-18] and is a subject of intense research activity in soft matter physics [17]. Some soft glassy materials (colloidal suspensions, adhesive emulsions) exhibit permanent (steady-state) SB, which is complemented by a strong shear-history-dependent rheological behavior [15-33]. Other simple yield stress fluids (nonadhesive emulsions, polymer solutions, wormlike micelles, foams) show transient SB during the flow startup [34-36]. The occurrence of shear bands, their stability, the selection of their size, and the timescales involved in their development are nontrivial issues, which have motivated many theoretical studies [16,37-41]. It has been shown there that the existence of an unstable branch in the flow curve, where the shear stress decreases with the shear rate and the existence of nonlocal effects related to the microstructure of the glassy materials are two crucial factors to yield the rich dynamics of SB.

SB in granular materials is less observed $[9,10,42]$ and lacks a thorough understanding. Some studies $[4,43,44]$ observed static regions in cohesionless or cohesive granular materials flowing in configurations like wide-gap cylindrical or split bottom Couette shear cells and reported this phenomenon as SB. However, this phenomenon is nothing but a usual solid-liquid coexistence [15], caused by the presence of heterogeneous shear stress profiles and a yield criterion. Here we exhibit true SB in cohesive granular media in the plane shear configuration, where the stress distribution is homogeneous, and present a detailed analysis.

The paper is organized as follows. Section II presents the details of the geometry and numerical simulations. Section III contains simulation results and is divided into five subsections. Section III A gives the preliminary rheological data, showing the evidence of two different flow regimes: the homogeneous flow regime at high inertial numbers and the shear-banded flow regime at low inertial numbers. Section III B comprises the analysis of the rheology of homogeneous flows. Section IIIC reports the crucial features of SB. Section III D shows the evidence of a shear-weakening rheological branch, which is behind the flow heterogeneity. Section III E includes the measurements of the (flow) starting and (flow) stopping friction coefficients from stress-imposed simulations. Section IV contains the theoretical analysis of SB based on a nonlocal rheological model [45], where a nonmonotonic rheological curve is introduced. Finally, conclusions and perspectives are detailed in Sec. V.

\section{DISCRETE ELEMENT METHOD (DEM) SIMULATIONS}

The material used in the simulations is an assembly of frictional, inelastic, cohesive spherical grains of (mean) mass $m$ and (mean) diameter $d$ with a size polydispersity of $20 \%$. The grains experience contact forces upon overlap, which are computed using the Hookean spring-dashpot model with a frictional slider and a Johnson-KendallRoberts-like [46], yet nonhysteretic, short-range, adhesive force model [7]; see Ref. [13] for more details. The normal contact force between two grains $i$ and $j$ has three contributions: (i) an elastic contribution $N_{i j}^{\mathrm{el}}=-k_{n} \delta$, where $k_{n}$ is the normal stiffness and $\delta$ is the normal overlap, (ii) a viscous contribution $\boldsymbol{N}_{i j}^{\mathrm{vis}}=-\gamma_{n} m_{\mathrm{eff}} \boldsymbol{c}_{i j}^{n}$, where $\boldsymbol{c}_{i j}^{n}$ is the normal relative velocity, $m_{\text {eff }}=m_{i} m_{j} /\left(m_{i}+m_{j}\right)$ is the effective mass, and $\gamma_{n}$ is the normal damping coefficient, and (iii) an adhesive contribution $N_{i j}^{\text {ad }}=\sqrt{4 k_{n} N_{c} \delta}$, which is assumed to be proportional to the area of contact, where $N_{c}$ is the minimum pull-off force necessary for the detachment of two contacting grains, as evident from Fig. 1(a). Note that the adhesive force vanishes when two grains are not in contact $[47,48]$, unlike in wet capillary bridges. The tangential contact force has only an elastic contribution $\boldsymbol{T}_{i j}^{\mathrm{el}}=-k_{t} s$, where $k_{t}$ is the tangential stiffness and $s$ is the relative tangential overlap from the beginning of a contact. It is set as $\boldsymbol{T}_{i j}=\mu_{p}\left(\boldsymbol{N}_{i j}^{\mathrm{el}}+\boldsymbol{N}_{i j}^{\mathrm{vis}}\right)\left(\mu_{p}\right.$ is the interparticle friction coefficient) when sliding of the contact occurs. The total contact force is the sum of the normal and tangential forces. Equations of motion are solved using the Verlet algorithm with a (dimensionless) time step $d t \approx 3 \times 10^{-5}$, which is nearly $1 / 70$ of the typical collision time for a pair of equal-sized cohesionless grains.

The three-dimensional plane shear cell [shown in Fig. 1(b)] comprises two rough walls of the cross-sectional area $A=20 d \times 20 d$ and height $1.8 d$, made of randomly glued grains; the choice of such boundaries rules out the possibility of wall slip. The system has periodic boundaries in the flow $(x)$ and vorticity $(y)$ directions. Gravity is absent in the system. The grains are first arranged in a simple cubic lattice in the shear cell and are assigned random velocities. 


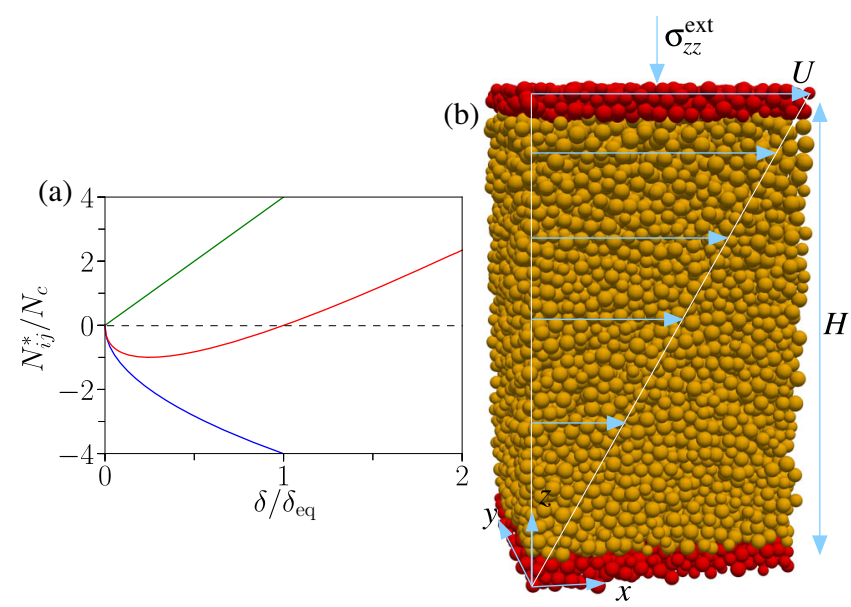

FIG. 1. Numerical model. (a) The normalized nonviscous normal contact forces $\left(N_{i j}^{*} / N_{c}\right)$ as a function of the normalized overlap $\delta / \delta_{\text {eq }}$ : elastic component $N_{i j}^{\mathrm{el}}$ (green line), adhesive component $N_{i j}^{\text {ad }}$ (blue line), and the sum of the two $N_{i j}^{\text {tot }}$ (red line). $N_{c}$ is the pull-off force, and $\delta_{\text {eq }}$ is the equilibrium overlap, as in Ref. [13]. (b) A simulation snapshot, showing a homogeneous flow in the normal-stress-imposed plane shear cell. Red particles constitute the rough boundaries.

Three different shearing protocols are then followed, maintaining a constant confining vertical stress $\sigma_{z z}^{\text {ext }}$ on the top wall and keeping the bottom wall fixed. In the first one, named here as steady shear rate up sweep, the shear rate is gradually increased by increasing the top wall velocity $U$ in a small step $\Delta U$ and allowing the system to reach a steady state after each increment. A steady state is signified by a constant gap $H$ between the two walls and a constant vertical stress $\sigma_{z z}$ across the gap, equal to $\sigma_{z z}^{\text {ext }}$, three different numbers of grains yield different $H: H / d \approx 8$ for $n=2400, H / d \approx 15$ for $n=4800$, and $H / d \approx 34$ for $n=12000$ at a low shear rate for the largest value of $N_{c}$ used (given below). In the second protocol, named here the shear rate jump, a particular shear rate is directly reached by moving the top wall with a constant velocity $U$, skipping the intermediate steady states at lower shear rates. After reaching a sufficiently high shear rate, the third protocol, named here the steady shear rate down sweep, is followed where the shear rate is gradually decreased by decreasing $U$ in a similar step $\Delta U$ and allowing the system to reach a steady state after each decrement. The steady velocity $\left[v_{x}(z)\right]$, volume fraction $[\phi(z)]$, and stress $\left[\tau_{x z}(z), \sigma_{z z}(z)\right]$ profiles are recorded in bins of $20 d$ (in $x) \times 20 d$ (in $y$ ) $\times 1 d$ (in $z$ ) over a strain window of 2 . The inertial number $I(z)$ and the friction coeffcient $\mu(z)=\tau_{x z}(z) / \sigma_{z z}(z)$ are extracted from these raw data. The data are averaged over four sets and are reported in dimensionless forms, considering $d$ as the length scale, $\left(m / \sigma_{z z}^{\text {ext }} d\right)^{1 / 2}$ as the timescale, and $\sigma_{z z}^{\text {ext }}$ as the stress scale. Some additional stress-imposed simulations are performed to measure the (flow) starting $\left(\mu^{\text {start }}\right)$ and (flow) stopping $\left(\mu^{\text {stop }}\right)$ friction coefficients, and the details are given below.
Unless otherwise specified, the model parameters used in the simulations are normal spring constant $k_{n} /\left(\sigma_{z z}^{\text {ext }} d\right)=$ $2 \times 10^{5}$, tangential spring constant $k_{t}=2 / 7 k_{n}$, and quality factor $Q=0.94\left(Q=\sqrt{k_{n} / m} / \gamma_{n}\right.$ is a function of the restitution coefficient in the cohesionless case [13]; the higher the $Q$, the lesser the inelastic dissipation), sliding friction coefficient $\mu_{p}=0.5$, and various adhesion $N_{c} /\left(\sigma_{z z}^{\text {ext }} d^{2}\right)$ from 0 (cohesionless) to 17.1 .

\section{RESULTS}

\section{A. Typical flow curves and preliminary observations}

Figure 2 shows the two classical rheological curves, as generally used for the characterization of the rheology of dry granular materials, namely the macroscopic friction coefficient $\mu$ and the volume fraction $\phi$ as a function of the

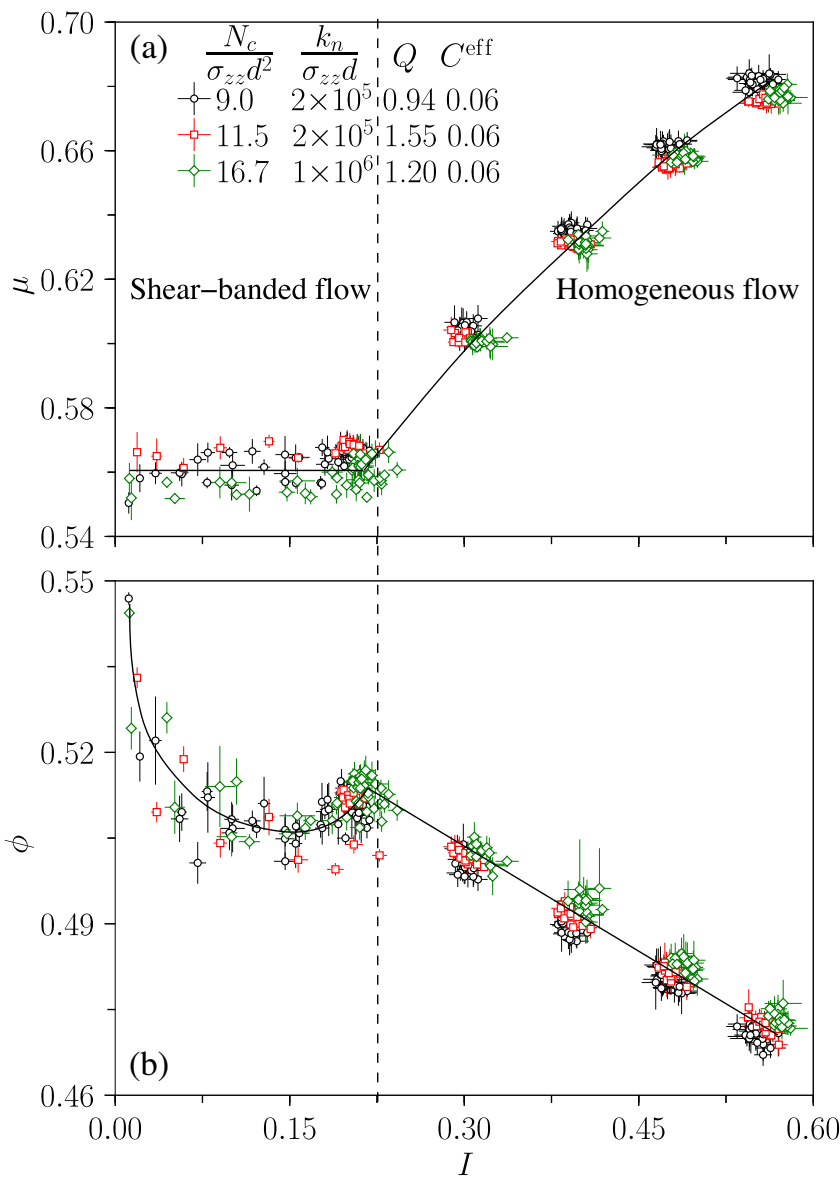

FIG. 2. Typical flow curves. Variation of (a) the friction coefficient $\mu$ and (b) the volume fraction $\phi$ with the inertial number $I$ for "effective" cohesion number $C^{\text {eff }}=0.06$ for three different sets of model parameters $\left(N_{c}, k_{n}, Q\right)$. Data are obtained using $n=12000$ grains during the steady shear rate up sweep. Error bars show the standard deviations over four sets. The solid lines are guides to the eye. The dashed line, corresponding to a critical inertial number $I_{c}$, demarcates the regime of steady, homogeneous flows from the regime of shear-banded flows. 
inertial number $I$ for three different sets of the model parameters $\left(N_{c}, k_{n}, Q\right)$. The curves are made of the local data for different $U$, obtained during the steady shear rate up sweep. Consider first the black curves, which are obtained using one set of parameters. At first sight, two different behaviors are evident. On the right-hand side of the dashed line, denoting a critical inertial number $I_{c} \approx 0.23, \mu$ increases, and $\phi$ decreases with increasing $I$, which are typical trends reported for the cohesive as well as dry cases [4,5,7-12,49-51]. We call this regime the homogeneous flow regime as the material here is homogeneously sheared, resulting in nearly constant $I, \mu$, and $\phi$ across the gap, as apparent from the data clouds. On the left-hand side of the dashed line, a plateau in the value of $\mu$ is observed, together with a nonmonotonic behavior of $\phi(I)$. We call this regime the shear-banded flow regime as the material here is inhomogeneously sheared across the gap, resulting in shear-banded velocity $v_{x}(z)$ and nonuniform $I(z)$ and $\phi(z)$ profiles. We discuss in detail below the features of these two flow regimes for various adhesion. Before that, we must note that $k_{n}$ and $Q$ also influence the rheology, as found in the inclined plane [13]. Following our earlier approach [13], we, therefore, define the effective adhesion $N_{c}^{\text {eff }}$ and the effective cohesion number $C^{\text {eff }}$ as

$$
\begin{gathered}
N_{c}^{\mathrm{eff}}=N_{c}\left[\left(\frac{N_{c}}{k_{n} d}\right)^{a} \frac{1}{Q^{b}}\right], \\
C^{\mathrm{eff}}=\frac{N_{c}^{\mathrm{eff}}}{\sigma_{z z} d^{2}},
\end{gathered}
$$

where $a$ and $b$ are unknown constants to be determined. For this purpose, we obtain various $\mu(I)$ and $\phi(I)$ curves (following the same protocol) for different values of $\left(N_{c}, k_{n}, Q\right)$. Considering first the subset of $\mu(I)$ and $\phi(I)$ curves for a constant $Q$, we detect the ones which yield good collapses and determine $a$ such that $C^{\text {eff }}$ [Eq. (2)] is the same for those. Similarly, considering the subset of $\mu(I)$ and $\phi(I)$ curves for a constant $k_{n}$, we detect the ones which yield good collapses and determine $b$ such that $C^{\text {eff }}$ is the same for those. We show in Fig. 2 such a data collapse for a given $C^{\text {eff }}=0.06$ with $a=0.5$ and $b=0.7$, resulting from different $\left(N_{c}, k_{n}, Q\right)$. This validates the newly proposed constitutive relations involving $C^{\text {eff }}$. Note that the value of $a=0.5$ determined here is the same as in the inclined plane study, but $b$ are different in the two cases: $b=0.7$ in the present case and $b=0.25$ in the inclined plane.

\section{B. Rheology of homogeneous flows}

We first analyze the rheology of homogeneous flows observed at high $I$. Figure 3 shows the variation of $\mu$ and $\phi$ with $I$ for different values of $C^{\text {eff }}$. For a given $I, \mu$ increases, and $\phi$ decreases with increasing $C^{\text {eff }}$, which agrees with previous studies [7-11,13]. Note that the range of inertial number for which homogeneous flows are observed seems to decrease with increasing $C^{\text {eff }}$, a point which is discussed later.

\section{Shear-banded flow regime}

We discuss here in detail the salient features of the shearbanded flow regime observed at low I. Figure 4(a) shows velocity profiles $v_{x}(z)$ obtained for sufficiently small wall velocities $U$ for an intermediate value of adhesion $\left(C^{\text {eff }}=0.1\right)$ during the steady shear rate up sweep. A shear band is first seen to appear near the top wall at a small $U$, which gradually expands in size when increasing $U$, until a homogeneous flow develops at a higher $U$. The local shear rate $\dot{\gamma}_{\text {loc }}$ in the shear band remains roughly the same when increasing $U$ [Fig. 4(a)], in accordance with the so-called lever rule [15] for soft glassy materials. However, when analyzed in more detail, a more complex scenario emerges than the one depicted in Fig. 4(a), which is highlighted below.
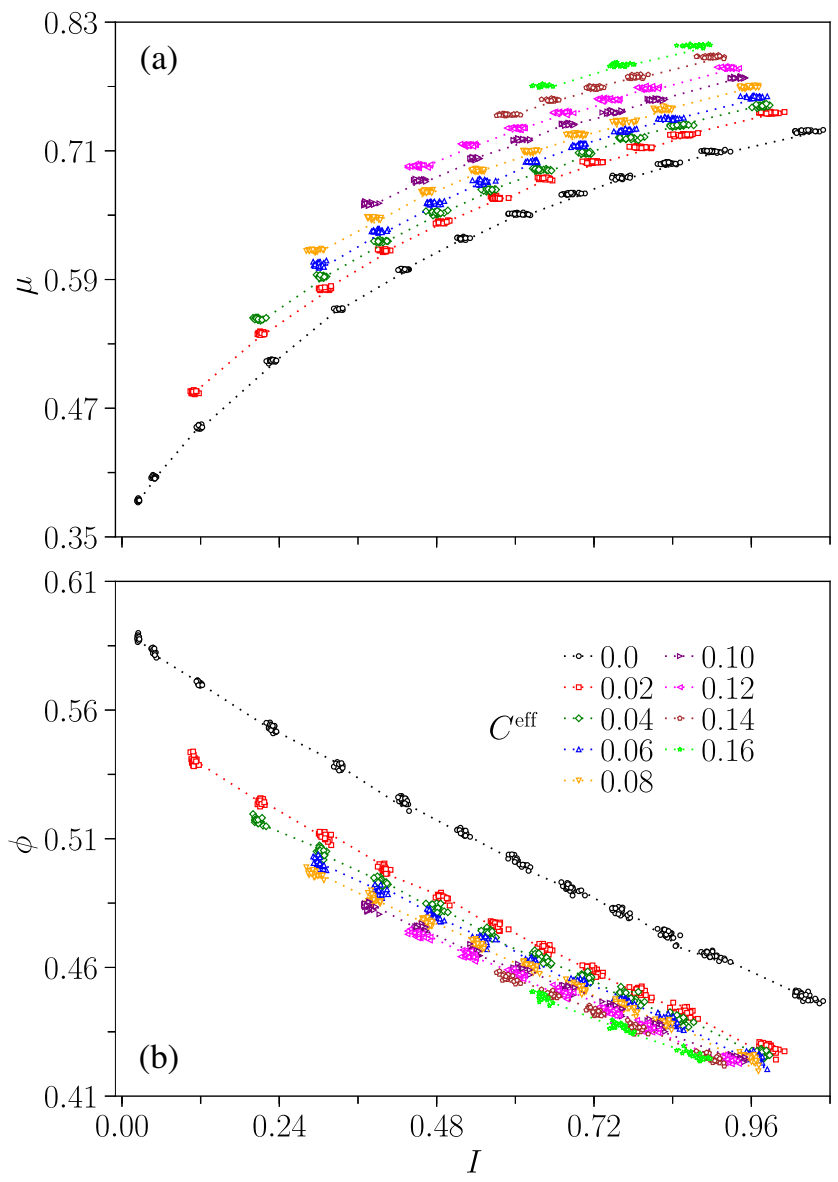

FIG. 3. Rheology of homogeneous flows. Variation of (a) the friction coefficient $\mu$ and (b) the volume fraction $\phi$ with the inertial number $I$ for different values of the effective cohesion number $C^{\text {eff }}$. Data are obtained using $n=12000$ grains during the steady shear rate up sweep. Dotted lines are guides to the eye. 

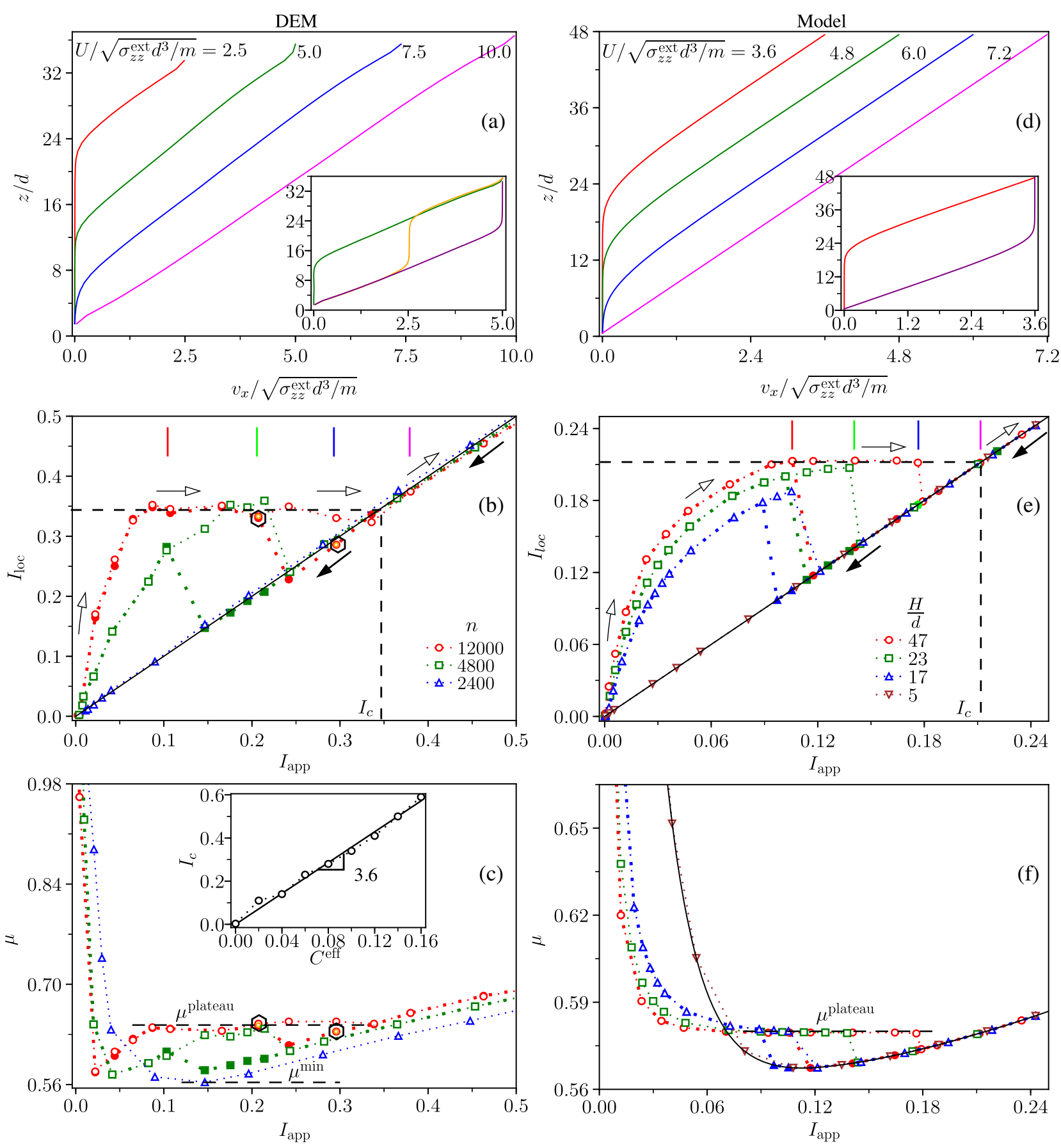

FIG. 4. Shear-banded flow regime in discrete (left-hand column) and continuum (right-hand column) simulations. (a) Evolution of the steady shear-banded velocity profile $v_{x}(z)$ with increasing $U$, following the steady shear rate up sweep, for $C^{\text {eff }}=0.1$ and $(n=12000$. Inset in (a): velocity profiles, obtained following different shearing protocols; (left) the steady shear rate up sweep, (middle) the shear rate jump, (right) the steady shear rate down sweep. (b) The local inertial number $I_{\mathrm{loc}}$ as a function of the apparent inertial number $I_{\text {app }}$ for different system sizes $(n)$ for $C^{\text {eff }}=0.1$. Data for very high $I_{\text {app }}$ are not shown for clarity. Data are extracted from steady $v_{x}(z)$, obtained following the steady shear rate up sweep (open symbols) and the steady shear rate down sweep (filled symbols). Orange hexagons enclosed in black hexagons correspond to the data obtained following the shear rate jump. Vertical lines in different colors, aligning with circular symbols, correspond to the data for the velocity profiles of the respective color shown in (a). The arrows give the directions for the sweep. The dashed lines indicate the value of the critical inertial number $I_{c}$ above which a fully developed flow is established for the largest system $(n=12000)$. (c) Friction coefficient $\mu\left(I_{\text {app }}\right)$ for the same simulations as in (b). The plateau in $\mu$, $\mu^{\text {plateau }}$, and the minimum of the intrinsic rheological curve, $\mu^{\text {min }}$, are indicated by dashed lines. Inset in (c): $I_{c}$ as a function of $C^{\text {eff }}$. The solid line is a linear least-squares fit to the data with a slope of 3.6. (d)-(f) Similar plots as in (a)-(c) from the continuum simulations, obtained for $\beta=1$, introducing the intrinsic rheological curve $\mu_{\text {int }}(I)$ [solid line in (f)] measured in DEM for $C^{\text {eff }}=0.1, n=2400$. 
Location of the shear band.-The location of the shear band at a given $U$ is not always fixed near the moving top wall as shown in Fig. 4(a). However, it is highly dependent on the initial condition and the shearing protocol followed, as manifested in the inset of Fig. 4(a). The band at $U / \sqrt{\sigma_{z z}^{\text {ext }} d^{3} / m}=5.0$ is located near the fixed bottom wall (purple) during the steady shear rate down sweep, starting from a homogeneously presheared sample. Moreover, the band splits into two, one localizing near the top and the other near the bottom, when $U / \sqrt{\sigma_{z z}^{\text {ext }} d^{3} / m}=5.0$ is reached following the shear rate jump, starting from the unsheared sample. However, the value of $\dot{\gamma}_{\text {loc }}$ remains the same in all three cases.

Domain of existence of the shear band.-To define precisely the domain of existence of the shear band, we consider a wide range of $U$. For each $U$, we extract the local shear rate $\dot{\gamma}_{\text {loc }}$ from a linear least-squares fitting of $v_{x}(z)$ data over the shear zone.Then we plot in Fig. 4(b) the local inertial number $I_{\mathrm{loc}}=\dot{\gamma}_{\mathrm{loc}} d / \sqrt{\sigma_{z z} / \rho_{p}}$ as a function of the imposed apparent inertial number $I_{\text {app }}=\dot{\gamma}_{\text {app }} d / \sqrt{\sigma_{z z} / \rho_{p}}$, where $\dot{\gamma}_{\text {app }}=U / H$. Data for three different system sizes $n$, corresponding to different protocols, steady shear rate up sweep (open symbols) and steady shear rate down sweep (filled symbols), are given. Consider first the case of the largest system $(n=12000)$ during the up sweep (red open symbols). For very small $I_{\text {app }}$, we surprisingly observe homogeneous flows, not SB, as indicated by the $I_{\text {loc }}=I_{\text {app }}$ relation (the diagonal line). Then with increasing $I_{\text {app }}$ until $I_{\text {app }} \approx 0.09$, we notice anomalous $\mathrm{SB}$, for which the band expands with a variable $I_{\text {loc }}$ with increasing $I_{\text {app }}$, disobeying the lever rule. With increasing $I_{\text {app }}$ above $I_{\text {app }} \approx 0.09$, the band keeps on expanding, maintaining a constant $I_{\text {loc }}$, which is the typical behavior, as per the lever rule, shown in Fig. 4(a). Finally, the flow becomes homogeneous, fully developed for $I_{\text {app }} \gtrsim 0.34$, which is taken as the critical inertial number $I_{c}$. The corresponding transition in the $\mu\left(I_{\text {app }}\right)$ rheology is shown in Fig. 4(c); data for very small $I_{\text {app }}$ are not given for clarity. For small values of $I_{\text {app }}, \mu$ is significantly higher than that given in Fig. 3(a) for homogeneous flows. With increasing $I_{\text {app }}, \mu$ decreases sharply before reaching a plateau for $I_{\text {app }} \gtrsim 0.09$, similar to the one in $I_{\mathrm{loc}}$. A similar plateau in the shear stress value is often considered as the signature of SB for soft glassy materials [16,17]. The behavior of $\mu\left(I_{\text {app }}\right)$ for $I_{\text {app }} \gtrsim 0.34$ is then the same as shown in Fig. 3(a). A similar overall scenario is noticed for other values of $C^{\text {eff }}$. However, the domain of $\mathrm{SB}$, delineated by $I_{c}$, increases with increasing $C^{\text {eff }}$, as manifested in the inset of Fig. 4(c). This implies that the stronger the adhesion, the higher the tendency toward shear localization, which agrees with a similar conclusion made for other soft glassy materials [22,25,27,28,30,31]. Moreover, the value of the plateau in $\mu, \mu^{\text {plateau }}$, increases near linearly with increasing $C^{\text {eff }}$, as shown in Fig. 6(c).
Manifestation of hysteresis.-The above description concerned the data obtained during the steady shear rate up sweep. When the down sweep of the shear rate (filled symbols) is performed starting from the homogeneously presheared sample at high $I_{\text {app }}$, the system manifests hysteresis [Figs. 4(b) and 4(c)]. SB appears for $I_{\text {app }} \lesssim 0.21$, exhibiting bistability for $0.21 \lesssim I_{\text {app }} \lesssim 0.34$, which means that the system may exhibit either a homogeneous or a shear-banded flow depending on the initial condition over this range of $I_{\text {app }}$. For example, the system shows a homogeneous flow at $I_{\text {app }} \approx 0.30$ (orange hexagon) during the shear rate jump. A similar hysteresis in the localization of shear bands has been reported in the literature for soft glassy materials [29,31,32].

Influence of the system size.-The above characterization of SB is given for a large system size $(n=12000)$. Considering a smaller system size $(n=4800)$ (green symbols), a similar scenario of SB appears, with the existence of a plateau for $I_{\mathrm{loc}}$ and $\mu$ and the manifestation of hysteresis [Figs. 4(b) and 4(c)]. However, in this case, SB occurs over a smaller range of $I_{\text {app }}$. In other words, homogeneous flows are observed over a wider range. Ultimately, for the smallest system size $(n=2400)$ (blue symbols), SB is seen to be entirely suppressed. In such small systems, corresponding to a layer thickness $H / d \approx 8$, the shear rate is observed to be uniform across the gap, as given by $I_{\mathrm{loc}}=I_{\text {app }}$ [Fig. 4(b)]. $\mu$ no longer exhibits a plateau but shows a nonmonotonic variation with increasing $I_{\text {app }}$ with a minimum $\mu^{\text {min }}$ [Fig. 4(c)]. Interestingly, the value of $\mu_{\min }$ is lower than the value of $\mu^{\text {plateau }}$ observed in large systems. The suppression of SB in a small system is also observed for other values of $C^{\text {eff }}$ and is also reported for soft glassy materials [30,31]. In Sec. IV, we reveal that the gradual disappearance of the shear band with decreasing system size is due to increasing nonlocal effects.

\section{Shear-weakening rheological branch}

Employing the data for a small system $(n=2400)$, it is then possible to obtain the intrinsic $\mu(I)$ and $\phi(I)$ rheological curves for all $C^{\text {eff }}$. Before that, we characterize these curves for a given $C^{\text {eff }}=0.06$ in Figs. 5(a) and 5(b). Consider first the black curves, which are obtained using one set of parameters. Both the curves are strikingly nonmonotonic, exhibiting a strong shear-weakening behavior $-\mu$ decreases, and $\phi$ increases remarkably with increasing $I$ until $I \approx 0.12$. Beyond this value of $I, \mu$ increases, and $\phi$ decreases, exhibiting a usual shear-strengthening behavior. We have checked that these constitutive curves change within $5 \%$ when considering even a smaller system size $(n=1600)$. The existence of such shear weakening rheological branch is commonly assumed to give rise to flow heterogeneity $[16,26,30,37,38,40,41]$ in soft glassy materials. 

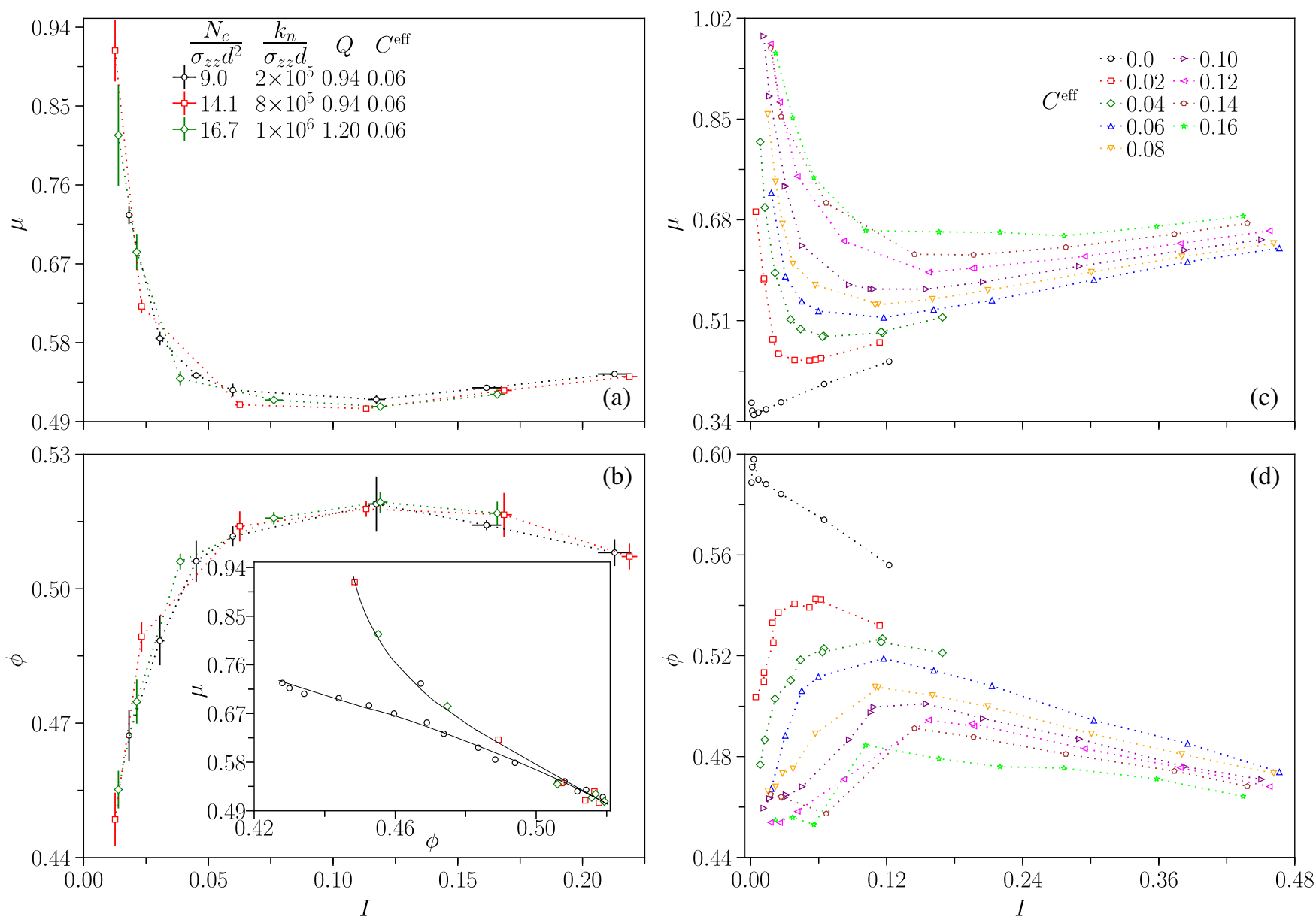

FIG. 5. Nonmonotonic intrinsic rheological curves. (a) The friction coefficient $\mu$ and (b) the volume fraction $\phi$ as a function of the inertial number $I$ for effective cohesion number $C^{\text {eff }}=0.06$ for three different sets of model parameters $\left(N_{c}, k_{n}, Q\right)$. Inset: $\mu$ as a function of $\phi$ for the same data as given in (a) and (b), except for the first set of parameters (black circles), for which additional data are included for high $I$. Solid lines are guides to the eye. (c) $\mu(I)$ and (d) $\phi(I)$ for different values of $C^{\text {eff }}$. Data are obtained for different $U$ during the steady shear rate up sweep using $n=2400$. Error bars in (a) and (b) show the standard deviations over four sets.

We showed in our previous study [13] that the initiation of flow down an inclined plane and the (flow) starting friction coefficient $\mu^{\text {start }}=\mu(I=0)$ are controlled by the cohesion number $C$, not the effective cohesion number $C^{\mathrm{eff}}$, unlike in the dynamic case. It is then compelling to know if the rheology in the shear-weakening branch, adjacent to the static rheological branch, is also controlled by $C$. However, we have checked that this is not true, as changing $k_{n}$ and $Q$ modify the rheological curves. The rheology in the shear weakening branch is indeed controlled by the same $C^{\text {eff }}$ [Eq. (2)] as $\mu(I)$ and $\phi(I)$ for different $\left(N_{c}, k_{n}, Q\right)$, resulting in the same $C^{\text {eff }}$, collapse together [Figs. 5(a) and 5(b)]. Looking at Figs. 5(a) and 5(b), one can also wonder if a unique relation between $\mu$ and $\phi$ exists considering both the shear-weakening and shear-strengthening branches together. Figure 5(b) (inset) reveals that it is not true. We have not been able to put in evidence the expected breakdown of this scaling for $I \rightarrow 0$, as we notice that the simulations for very small inertial numbers in the presence of cohesion require a very small time step for ensuring convergence. We restrict our study to $I>0.01$ to stay within a reasonable computing time.

Figures 5(c) and 5(d) show the intrinsic $\mu(I)$ and $\phi(I)$ curves for different values of $C^{\text {eff }}$. With increasing $C^{\text {eff }}$, the minimum friction coefficient $\mu^{\min }$ and the corresponding maximum volume fraction $\phi^{\text {max }}$ are noted to increase and decrease, respectively [see Fig. 6(c)]. Moreover, the minimum $I, I_{\min }$, for which $\mu=\mu^{\min }$ (or $\phi=\phi^{\max }$ ) also seems to increase with increasing $C^{\text {eff }}$. A nonmonotonicity, although much smaller than that for the cohesive case, is also observed for the cohesionless case [52,53] in both plots. For such small nonmonotonicity, we have not observed any SB even in our largest system $(n=12000, H / d \approx 29)$. However, we have checked that SB tends to appear in the cohesionless case 

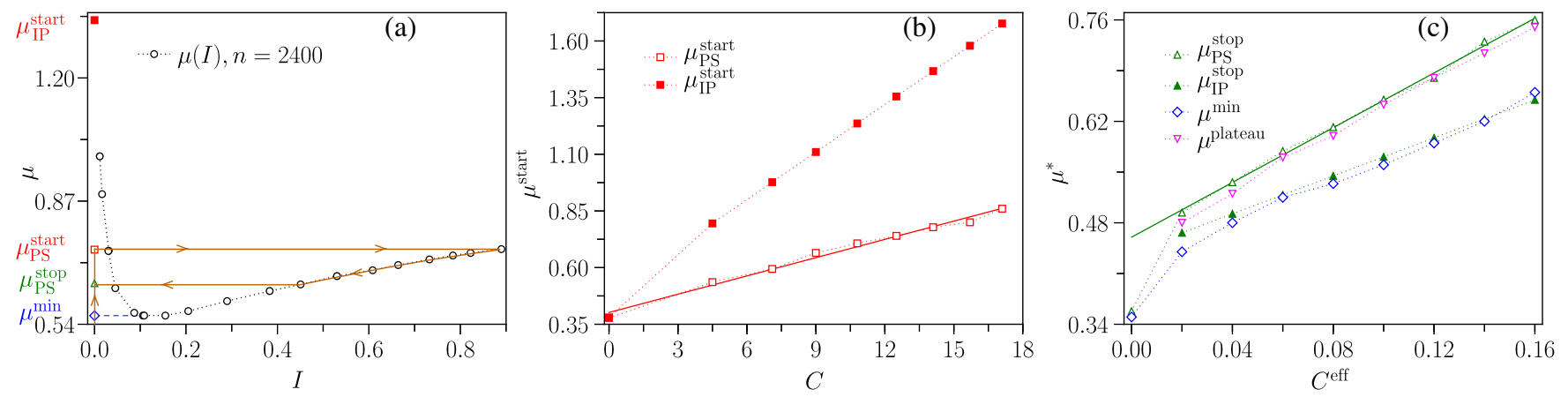

FIG. 6. Stress-imposed rheological measurements. (a) The starting $\mu_{\mathrm{PS}}^{\text {start }}$ and the stopping $\mu_{\mathrm{PS}}^{\text {stop }}$ friction coefficients for $C^{\text {eff }}=0.1$, measured using the plane shear cell, along with the intrinsic $\mu(I)$ rheological curve, measured from shear-rate-imposed simulations. The brown line depicts the flow starting and stopping paths. The dashed line indicates $\mu^{\mathrm{min}}$. The starting friction coefficient $\mu_{\mathrm{IP}}^{\text {start }}$, measured using the inclined plane, is also given. (b) $\mu^{\text {start }}$ as a function of the cohesion number $C$ for both the shear cell and the inclined plane (data from Ref. [13]). (c) Stopping $\mu_{\mathrm{PS}}^{\text {stop }}$, plateau $\mu^{\text {plateau }}$, minimum $\mu^{\text {min }}$ friction coefficients measured using the shear cell, and $\mu_{\mathrm{IP}}^{\text {stop }}$ measured using the inclined plane (data from Ref. [13]) as a function of the effective cohesion number $C^{\text {eff }}$. The solid lines in (b) and (c) are the fits of the Mohr-Coulomb failure equation.

once the system size is sufficiently large $(n=16000$, $H / d \approx 38$ ) at very small $I_{\text {app }}$. The rheology of cohesionless grains is usually not probed in such a large system. This fact a posteriori explains why SB is usually not reported for cohesionless grains.

\section{E. Stress-imposed simulations}

Utilizing shear-rate-imposed simulations in the previous section, we were able to extract the rheological curves. The (flow) starting $\mu^{\text {start }}$ and the (flow) stopping $\mu^{\text {stop }}$ friction coefficients [Fig. 6(a)] are two additional parameters of interest from the rheological perspective. For determining these, we perform shear-stress-imposed simulations, where a shear stress $\tau$ is imposed on the top wall instead of $U$. The measurements are carried out for different $\left(N_{c}, k_{n}, Q\right)$. We first obtain a static random configuration by gradually stopping the flow from a homogeneously sheared configuration in the shear-rate-imposed simulations. Then we impose $\tau$ on the top wall to shear the material and increase it gradually until the wall starts moving at $\tau=\tau^{\text {start }}$. The flow is then continued until a steady state is reached. The value of $\tau$ is then gradually lowered until the wall stops moving at $\tau=\tau^{\text {stop }} . \mu^{\text {start }}$ and $\mu^{\text {stop }}$ are then computed as $\mu^{\text {start }}=\tau^{\text {start }} / \sigma_{z z}$ and $\mu^{\text {stop }}=\tau^{\text {stop }} / \sigma_{z z}$.

Starting friction coefficient.-Before giving the values of $\mu^{\text {start }}$ for different adhesion, we confirm in this study also that it depends only on the interparticle adhesion $N_{c}$, not on the material properties $\left(k_{n}, Q\right)$, as found in the inclined plane study [13]. Hence, $\mu_{\mathrm{PS}}^{\text {start }}$ is given as a function of $C$ in Fig. 6(b), along with those measured from the inclined plane, using $\mu_{\mathrm{IP}}^{\text {start }}=0.48+0.07 C[13] . \mu_{\mathrm{PS}}^{\text {start }}$ is seen to increase linearly with increasing $C$, following the cohesive Mohr-Coulomb model (solid line). $\mu_{\mathrm{PS}}^{\text {start }}$ for the plane shear is significantly smaller than $\mu_{\mathrm{IP}}^{\text {start }}$ for the inclined plane, except for the cohesionless case, for which these two values are identical. This anomaly may arise from different stress distributions in the two geometries- the stress distribution is homogeneous in the shear cell but inhomogeneous with the highest stress values at the bottom in the inclined plane. This difference in stress distribution affects the scenario of yielding. In the inclined plane, the yield criterion is most likely satisfied first at the bottom only (for the cohesive grains), whereas it is simultaneously satisfied everywhere in the plane shear cell, which explains why $\mu_{\mathrm{PS}}^{\text {start }}<\mu_{\mathrm{IP}}^{\text {start }}$. Out of curiosity, we locate $\mu_{\mathrm{PS}}^{\text {start }}$ and $\mu_{\mathrm{IP}}^{\text {start }}$ in the intrinsic $\mu(I)$ curve for one $C^{\text {eff }}=0.1$ in Fig. 6(a) and observe that $\mu_{\mathrm{PS}}^{\text {start }}$ is strikingly lower than $\mu(I \approx 0.01)$, the maximum $\mu$ we have measured. Moreover, $\mu_{\mathrm{IP}}^{\text {start }}$ rather seems to well define the end of the intrinsic curve $[\mu(I=0)]$. A similar scenario is noticed for other $C^{\text {eff }}$.

Stopping friction coefficient.-Figure 6(c) gives $\mu_{\mathrm{PS}}^{\text {stop }}$ as function of $C^{\mathrm{eff}}$, along with the measurements from the inclined plane, using $\mu_{\mathrm{IP}}^{\text {stop }}=0.44+1.31 C^{\text {eff }}[13] . \mu_{\mathrm{PS}}^{\text {stop }}$ also increases linearly with increasing $C^{\text {eff }}$, following the cohesive Mohr-Coulomb model (solid line). In this case also, we notice some difference between the values of $\mu_{\mathrm{PS}}^{\text {stop }}$ and $\mu_{\mathrm{IP}}^{\text {stop }}$ with $\mu_{\mathrm{PS}}^{\text {stop }}>\mu_{\mathrm{IP}}^{\text {stop }}$. Locating again $\mu_{\mathrm{PS}}^{\text {stop }}$ in the intrinsic $\mu(I)$ curve in Fig. 6(a), we observe that $\mu_{\mathrm{PS}}^{\text {stop }}$ is surprisingly higher than the minimum of $\mu, \mu^{\min }$ [see Fig. 6(c) also], but $\mu_{\mathrm{IP}}^{\text {stop }} \approx \mu^{\min }$ [Fig. 6(c)]. Interestingly, the value of $\mu_{\mathrm{PS}}^{\text {stop }}$ is the same as the value of $\mu^{\text {plateau }}[$ Fig. 6(c)], as observed in the case of soft glassy materials [23,25,27]. These two facts signify that the stress- and shear-rate-imposed rheological measurements differ below $\mu_{\mathrm{PS}}^{\text {stop }}\left(I_{c}\right)$, as usually reported for soft glassy materials $[23,25,27]$. These also imply that SB appears before hitting the unstable branch below $I_{\min }$, as reported for soft glassy materials [16].

Note that the values of $\mu_{\mathrm{PS}}^{\text {start }}$ and $\mu_{\mathrm{PS}}^{\text {stop }}$ have no size dependency, unlike in the case of the inclined plane. 
The degree of hysteresis (nonmonotonicity), estimated as $\Delta \mu=\mu(I=0)-\mu^{\min }\left[\mu_{\mathrm{IP}}^{\text {start }}\right.$ is taken as $\left.\mu(I=0)\right]$ from Figs. 6(b) and 6(c), seems to increase with increasing adhesion, causing more prominent SB in cohesive granular media.

\section{NONLOCAL THEORETICAL MODEL FOR DESCRIBING SHEAR BANDING}

We have provided a comprehensive picture of SB and have revealed that many features of this phenomenon observed in our case are similar as in soft glassy materials. As mentioned in the introduction, several theoretical studies [16,37-41] reproduce the crucial features of SB observed with soft glassy material, by incorporating a non monotonic constitutive flow curve and a nonlocal stress diffusion term. Inspired by these studies, we take a similar approach and present a simple theoretical model based on the nonlocal model of Bouzid et al. [45], generally used for describing the rheology of dry granular flows, by introducing a nonmonotonic intrinsic rheological curve $\mu_{\text {int }}(I)$ (see the Appendix A). Since the work of Aranson and Tsimring [54], who developed a phenomenological nonlocal model with hysteresis based on a Ginzburg-Landau formalism, different theoretical continuum descriptions have been proposed to capture both the $\mu(I)$ rheology of dry granular flows and nonlocal effects [55]. The two most popular approaches are based either on the concept of fluidity [56] or on an extension in gradients of the inertial number [45]. Although differences exist between the two approaches [55,57], specifically in terms of stability [58], for the one-dimensional plane shear configuration of interest in this paper, they are equivalent. For the sake of simplicity, we adopt here the model proposed by Bouzid et al. [45], in which the implementation of intrinsic cohesive rheology is straightforward. The model stands on the following constitutive law, relating the local friction coefficient $\mu(z)=\tau_{x z}(z) / \sigma_{z z}(z)$ to the local inertial number $I(z)$ (assuming that all the flow properties vary only in the $z$ direction) as

$$
\mu=\mu_{\mathrm{int}}(I)\left(1-\beta^{2} \frac{\partial^{2} I / \partial z^{2}}{I}\right),
$$

where $\beta$ is a characteristic length, defining the range of the nonlocal effects and is typically of the order of few particle diameters $(\beta / d=1$ in the following). The nonlocality is embedded in the second term on the right-hand side, which incorporates the idea of the long-range dynamical cooperativity $[59,60]$ and helps the neighborhood of a flowing region to flow by reducing the friction whenever there exists a gradient of the inertial number. The equation is solved numerically using a finite difference scheme (see Appendix A) to obtain the steady velocity profiles $v_{x}(z)$ for different wall velocities $U$. No-slip conditions on the two boundary walls and a zero gradient of the inertial number across the interfaces of the boundaries are used as boundary conditions. Note that the nonlocal model is solved (see Appendix A), taking into account the inertia of the system, closely mimicking the discrete simulation methodology in terms of the time development of the flow.

Figure 4 presents the results obtained from the model alongside the discrete simulations results. Figure 4(d) shows the velocity profiles for different $U$ for a large system $(H / d=47)$, obtained following the steady shear rate up sweep. A shear band is seen to be localized near the top, as seen in the discrete simulations. The growth of the band, maintaining a constant local shear rate $\dot{\gamma}_{\text {loc }}$, with increasing $U$ is again similar as in the discrete simulations. The location of the band depends on the initial condition (inset) as in the discrete simulation-the band localizes near the bottom when the down sweep of the shear rate is performed. Figure 4(e) shows the local inertial number $I_{\mathrm{loc}}$ as a function of the apparent inertial number $I_{\text {app }}$ over a wide range. The appearance of homogeneous flows at very small $I_{\text {app }}$, anomalous SB at small $I_{\text {app }}, \mathrm{SB}$ obeying the lever rule at sufficiently higher $I_{\text {app }}$, and finally, the reappearance of homogeneous flows at higher $I_{\text {app }}$ are again similar as in the discrete simulations. The corresponding $\mu\left(I_{\text {app }}\right)$ are given in Fig. 4(f); the data for very small $I_{\text {app }}$, falling on the $\mu_{\text {int }}(I)$ curve, are not given for clarity. The plateau in the value of the friction coefficient above the minimum of the $\mu_{\text {int }}(I)$ curve is also reproduced successfully. This implies that the combination of a nonmonotonic flow curve and the nonlocality is enough for the selection of a well-defined friction coefficient for the shearbanded flow regime [37]. The features of the rheological hysteresis, depending on the initial condition and the shearing protocol followed, are also captured by the model [Figs. 4(e) and 4(f)]. Finally, the gradual suppression of SB and the corresponding transition in the rheology on decreasing the system size are also predicted by the model. Note that the measured $\mu\left(I_{\mathrm{app}}\right)$ for the smallest system size $(H / d=5)$ coincides with the intrinsic rheological curve $\mu_{\text {int }}(I)$ (solid line) introduced in the nonlocal model. This justifies a posteriori the use of a small system size in extracting the intrinsic rheological curve in the discrete simulations. The influence of $\beta$ on the results is shown in Fig. 8 . With increasing $\beta$, i.e., increasing nonlocal effects, a similar dynamics is noticed as on decreasing $H$. Thus, this simple nonlocal model remarkably reproduces crucial features of SB observed in the discrete simulations, and puts in evidence the roles of nonlocal effects and hysteresis (nonmonotonicity) in SB. However, it fails to make quantitative predictions. With $\mu_{\text {int }}(I)$ taken as an input, the only free parameter of the model is $\beta$. We observe that on varying $\beta$ over a reasonable scale from $\beta=1$ to 0.5 (see Fig. 8), the plateau of $I_{\text {local }}\left(I_{c}\right)$ and the plateau of $\mu$ ( $\left.\mu^{\text {plateau }}\right)$ 
(not shown) do not change and stay at lower levels compared to discrete element method (DEM) simulations.

\section{DISCUSSION AND CONCLUSIONS}

In this paper, we have numerically investigated the rheology of a model cohesive, frictional granular medium, in which a short-range interparticle adhesive force is active when two particles overlap. The material is sheared in a normal-stress-imposed plane shear cell over a wide range of shear rate, for various interparticle adhesion. Here we list the main results. First, the interparticle adhesion causes a systematic development of shear bands in the material, and the range of imposed shear rate over which the localization is observed increases with increasing adhesion. Second, the occurrence of SB is related to the existence of a nonmonotonic rheological curve, where the friction coefficient first decreases with increasing inertial number down to a minimum before increasing again at high inertial number. The existence of this intrinsically unstable shear-weakening branch is evidenced using a small system, where SB is stabilized. Third, the adhesion influences these nonmonotonic constitutive curves through an "effective" cohesion number, introduced in Ref. [13], combining the interparticle adhesion and the stiffness and inelasticity of the particles. Lastly, a theoretical model based on a nonlocal rheological model coupled with a nonmonotonic flow curve successfully reproduces the crucial features of SB observed in numerical simulations. It predicts the disappearance of shear bands in small systems or at very low shear rates. It captures the occurrence of rheological hysteresis during the upward and downward sweeps of the shear rate. It also yields a unique plateau in the friction coefficient above the minimum of the intrinsic rheological curve, although at a lower level compared to discrete simulations.

Interestingly, the scenario of SB exhibited for our cohesive, frictional granular material is qualitatively similar to that for other complex fluids. More prominent SB with increasing adhesion [22,25,27,28,30,31], rheological hysteresis depending on the initial configuration $[29,31,32]$, and a system size dependence [30,31] are also observed for soft glassy materials. Moreover, the two main ingredients of the theoretical analysis to capture the rich dynamics are also a nonmonotonic flow curve and nonlocality. However, the analogy between the two cases has to be drawn with care, considering that SB in our granular medium is investigated in the framework of pressure-imposed rheology, not the classical volume-imposed rheology.

An open question remains about the physical origin of the nonmonotonic flow curve in the cohesive granular media. In most complex fluids, the interplay between the shear-induced rejuvenation and the restructuration [18,26] is behind the existence of a critical shear rate and a shearweakening flow curve. However, for our cohesive granular material, it is not clear which time could play the role of a restructuring timescale, given the fact that a long-range attraction is absent and the material is athermal. Recently, Macaulay and Rognon [42] provided an interpretation of the nonmonotonic flow curve in the cohesive granular media from a microscale analysis. They showed that the friction coefficient comprises two parts: static and inertial. The static component, arising from balanced contact forces, decreases sharply from a high value with increasing inertial number, while the inertial one, arising from unbalanced forces, increases slowly from a low value with the inertial number. The resultant then ensures a minimum at an intermediate inertial number.

The existence of the hysteresis and a nonmonotonic flow curve in granular materials has been recently studied by Perrin et al. [61]. Using non-Brownian, viscous granular suspensions in which the interparticle friction could be tuned, they showed that the interparticle friction was

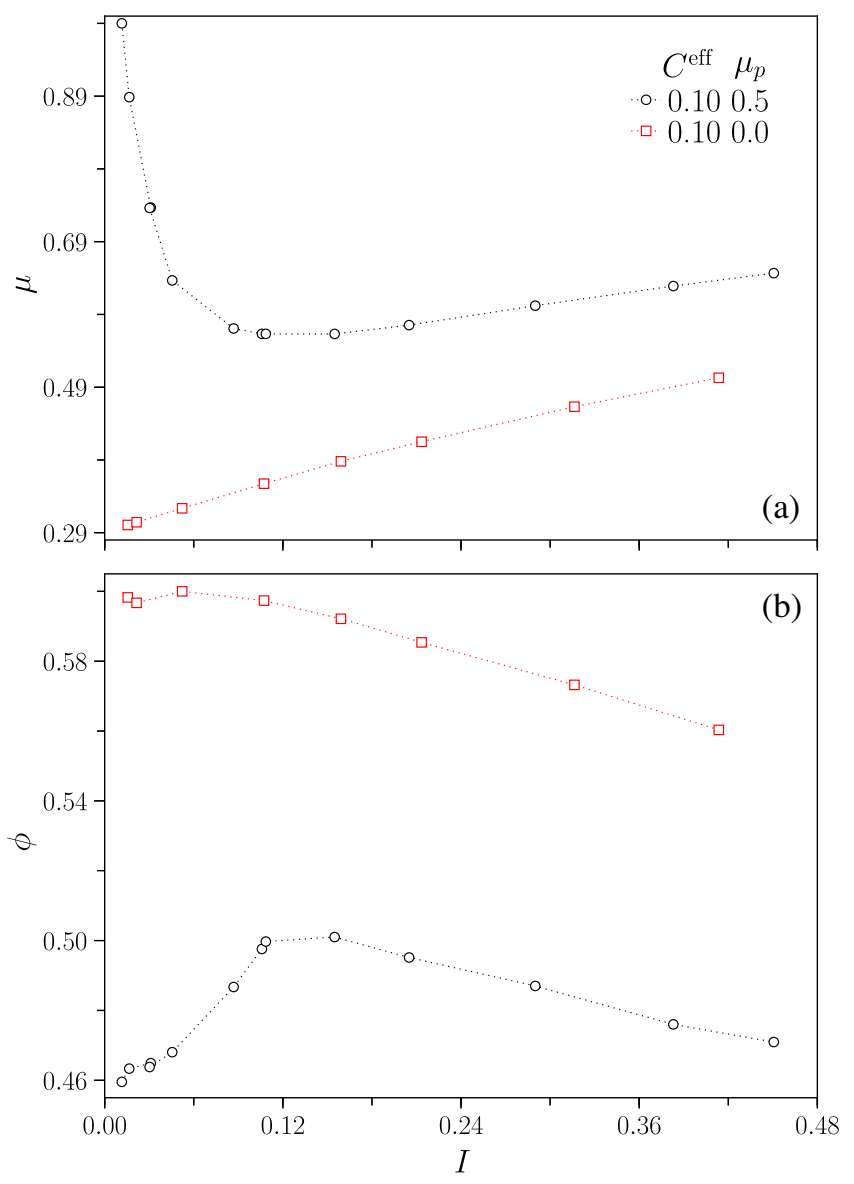

FIG. 7. Effect of the interparticle friction on the constitutive rheological curves. (a) $\mu(I)$ and (b) $\phi(I)$ for $\mu_{p}=0.5$ and $\mu_{p}=0$ for $C^{\text {eff }}=0.1$. 
necessary (contrary to inertia, which was not necessary) to obtain the hysteresis and a shear-weakening rheological branch. In the same line, we show here that the interparticle friction is indeed essential to introduce the nonmonotonicity in the rheological curve for our cohesive granular medium. No shear-weakening branch is obtained for a frictionless yet highly cohesive granular medium (Fig. 7). Hence, no SB is observed even in a large system. It will be interesting to know if the hysteresis observed in our cohesive granular medium has the same origin as in other cohesionless systems $[53,61]$ with the adhesion amplifying an already existing hysteresis, or if a different mechanism is involved.

Nevertheless, the existence of a strong hysteresis in cohesive granular media has important implications for the handling of powders in industries. In situations where the flow is unsteady and nonuniform, as encountered in industrial processes, instabilities and shear localization may lead to frequent clogging and jamming. The new insights into the rheology of cohesive granular materials provided by our study may suggest new roads for a better characterization of the "flowability" of powders.

\section{ACKNOWLEDGMENTS}

This work was supported by the ANR Grant No. ANR17-CE08-0017 under COPRINT (Cohesive Powders Rheology: Innovative Tools) project. The authors acknowledge Sébastien Manneville and Thibaut Divoux for a fruitful conversation.

\section{APPENDIX A: CONTINUUM SIMULATIONS USING THE NONLOCAL MODEL}

A nonmonotonic intrinsic rheological curve $\mu_{\text {int }}(I)$ of the following shape is introduced in the model:

$$
\mu_{\text {int }}(I)=c_{1}-\left(c_{1}-c_{2}\right)\left[1-\exp \left(-c_{3} I\right)\right]+c_{4} I^{c_{5}},
$$

where $c_{1}=1.36, c_{2}=0.56, c_{3}=53.75, c_{4}=0.43$, and $c_{5}=2$ are constants. This $\mu_{\text {int }}(I)$ closely approximates the intrinsic rheological curve for $C^{\text {eff }}=0.1$ [Fig. 6(a)] in the range $I \in(0,0.45)$. Combining Eqs. (3) and (A1) and approximating the derivative by a finite difference, we get

$$
\begin{aligned}
\tau_{x z}[i][t]= & \sigma_{z z}\left\{c_{1}-\left(c_{1}-c_{2}\right)\right. \\
& \left.\times\left[1-\exp \left(-c_{3} I[i][t]\right)\right]+c_{4} I[i][t]^{c_{5}}\right\} \\
& \times\left\{1-\frac{\beta^{2}}{I[i][t]} \frac{(I[i+1][t]-2 I[i][t]+I[i-1][t])}{\Delta z^{2}}\right\},
\end{aligned}
$$

where indices $t$ and $i$, pertaining to any quantity, represent the current time and the index of the spatial node, and $\Delta z$ is the spacing between two nodes. The simulation for a given small $U$ is initialized by taking a continuous shape of the velocity profile across the gap $H=(n-2) \Delta z$ ( $n$ is the number of nodes), for example, a slightly perturbed linear profile $v_{x}[i+1 / 2][0]=U / H(i-0.5) \Delta z+v^{\prime}$, where $v^{\prime}$ is a small random velocity. Shear rate is then calculated as $\dot{\gamma}[i][0]=\left(v_{x}[i+1 / 2][0]-v_{x}[i-1 / 2][0]\right) / \Delta z$, followed by inertial number $I[i][0]=\dot{\gamma}[i][0] d / \sqrt{\sigma_{z z} / \rho_{p}}$. After that, the shear stress $\tau_{x z}[i][0]$ is calculated from Eq. (A2). The following equation of motion is then solved (to get updated velocity) using the fourth-order Runge-Kutta method,

$$
\begin{aligned}
& v_{x}[i+1 / 2][t+d t] \\
& \quad=v_{x}[i+1 / 2][t]+\frac{\left(\tau_{x z}[i+1][t]-\tau_{x z}[i][t]\right)}{\rho \Delta z} d t,
\end{aligned}
$$

where $\rho=\phi \rho_{p}$ is the bulk density and $d t$ is the size of the time step. The whole algorithm is repeated until a steady state, indicated by a uniform shear stress profile, is reached. The simulation is done taking $\phi=0.55, \rho_{p}=(6 / \pi) m / d^{3}$, and $\Delta z / d=1.0$. A steady shear rate up sweep is then performed by increasing $U$ in a small step $\Delta U$, and a steady state is ensured after each increment. After reaching a sufficiently high apparent shear rate $\left(\dot{\gamma}_{\text {app }} / \sqrt{\sigma_{z z}^{\text {ext }} d / m} \approx\right.$ 0.25 ), a down sweep of the shear rate is performed by decreasing $U$ in a similar step. The whole set of simulations is repeated for different $\beta$ and $H$.

\section{APPENDIX B: EFFECT OF $\beta$}

Figure 8 shows the variation of the local inertial number $I_{\text {loc }}$ with the apparent inertial number $I_{\text {app }}$ for different values of $\beta$ for $H / d=29$; the trend is similar for other $H$ considered in the main text.

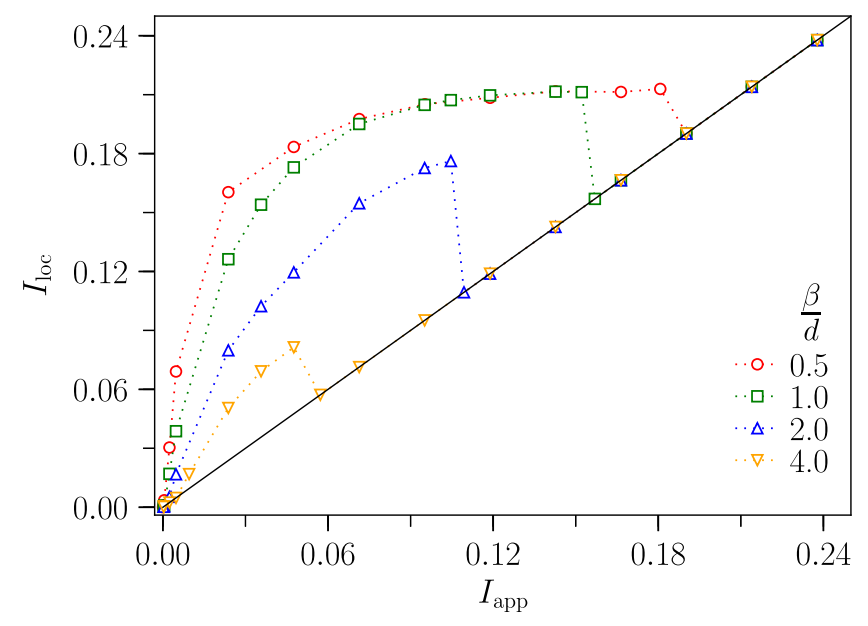

FIG. 8. The local inertial number $I_{\text {loc }}$ as a function of the apparent inertial number $I_{\mathrm{app}}$ for different values of $\beta$ for $H / d=29$. Data are extracted from steady $v_{x}(z)$, obtained following the steady shear rate up sweep. 
[1] M. V. V. Antequera, A. M. Ruiz, M. C. M. Perales, N. M. Munoz, and M. R. J.-C. Ballesteros, Evaluation of an Adequate Method of Estimating Flowability According to Powder Characteristics, Int. J. Pharm. 103, 155 (1994).

[2] D. Geldart, E. C. Abdullah, A. Hassanpour, L. C. Nwoke, and I. Wouters, Characterization of Powder Flowability Using Measurement of Angle of Repose, Chin. Particuol. 4, 104 (2006).

[3] H. Shi, R. Mohanty, S. Chakravarty, R. Cabiscol, M. Morgeneyer, H. Zetzener, J. Y. Ooi, A. Kwade, S. Luding, and V. Magnanimo, Effect of Particle Size and Cohesion on Powder Yielding and Flow, KONA Powder Part. J. 35, 226 (2018).

[4] G. MiDi, On Dense Granular Flows, Eur. Phys. J. E 14, 341 (2004).

[5] F. da Cruz, S. Emam, M. Prochnow, J. N. Roux, and F. Chevoir, Rheophysics of Dense Granular Materials: Discrete Simulation of Plane Shear Flows, Phys. Rev. E 72, 021309 (2005).

[6] P. Jop, Y. Forterre, and O. Pouliquen, A Constitutive Law for Dense Granular Flows, Nature (London) 441, 727 (2006).

[7] P. G. Rognon, J. N. Roux, D. Wolf, M. Naaïm, and F. Chevoir, Rheophysics of Cohesive Granular Materials, Europhys. Lett. 74, 644 (2006).

[8] P. G. Rognon, J. N. Roux, M. Naaim, and F. Chevoir, Dense Flows of Cohesive Granular Materials, J. Fluid Mech. 596, 21 (2008).

[9] S. Khamseh, J. N. Roux, and F. Chevoir, Flow of Wet Granular Materials: A Numerical Study, Phys. Rev. E 92, 022201 (2015).

[10] N. Berger, E. Azéma, J. Douce, and F. Radjai, Scaling Behaviour of Cohesive Granular Flows, Europhys. Lett. 112, 64004 (2015).

[11] M. Badetti, A. Fall, D. Hautemayou, F. Chevoir, P. Aimedieu, S. Rodts, and J. N. Roux, Rheology and Microstructure of Unsaturated Wet Granular Materials: Experiments and Simulations, J. Rheol. 62, 1175 (2018).

[12] T. T. Vo, S. Nezamabadi, P. Mutabaruka, J.-Y. Delenne, and F. Radjai, Additive Rheology of Complex Granular Flows, Nat. Commun. 11, 1476 (2020).

[13] S. Mandal, M. Nicolas, and O. Pouliquen, Insights into the Rheology of Cohesive Granular Media, Proc. Natl. Acad. Sci. U.S.A. 117, 8366 (2020).

[14] P. D. Olmsted, Perspectives on Shear Banding in Complex Fluids, Rheol. Acta 47, 283 (2008).

[15] G. Ovarlez, S. Rodts, X. Chateau, and P. Coussot, Phenomenology and Physical Origin of Shear Localization and Shear Banding in Complex Fluids, Rheol. Acta 48, 831 (2009).

[16] S. M. Fielding, Shear Banding in Soft Glassy Materials, Rep. Prog. Phys. 77, 102601 (2014).

[17] T. Divoux, M. A. Fardin, S. Manneville, and S. Lerouge, Shear Banding of Complex Fluids, Annu. Rev. Fluid Mech. 48, 81 (2016).

[18] D. Bonn, M. M. Denn, L. Berthier, T. Divoux, and S. Manneville, Yield Stress Materials in Soft Condensed Matter, Rev. Mod. Phys. 89, 035005 (2017).

[19] P. Coussot, J. S. Raynaud, F. Bertrand, P. Moucheront, J. P. Guilbaud, H. T. Huynh, S. Jarny, and D. Lesueur, Coexistence of Liquid and Solid Phases in Flowing Soft-Glassy Materials, Phys. Rev. Lett. 88, 218301 (2002).
[20] F. Varnik, L. Bocquet, J. L. Barrat, and L. Berthier, Shear Localization in a Model Glass, Phys. Rev. Lett. 90, 095702 (2003).

[21] L. Bécu, S. Manneville, and A. Colin, Yielding and Flow in Adhesive and onadhesive Concentrated Emulsions, Phys. Rev. Lett. 96, 138302 (2006).

[22] A. Ragouilliaux, G. Ovarlez, N. Shahidzadeh-Bonn, B. Herzhaft, T. Palermo, and P. Coussot, Transition from a Simple Yield-Stress Fluid to a Thixotropic Material, Phys. Rev. E 76, 051408 (2007).

[23] P. C. F. Møller, S. Rodts, M. A. J. Michels, and D. Bonn, Shear Banding and Yield Stress in Soft Glassy Materials, Phys. Rev. E 77, 041507 (2008).

[24] R. Besseling, L. Isa, P. Ballesta, G. Petekidis, M. E. Cates, and W. C. K. Poon, Shear Banding and Flow-Concentration Coupling in Colloidal Glasses, Phys. Rev. Lett. 105, 268301 (2010).

[25] A. Fall, J. Paredes, and D. Bonn, Yielding and Shear Banding in Soft Glassy Materials, Phys. Rev. Lett. 105, 225502 (2010).

[26] P. Coussot and G. Ovarlez, Physical Origin of ShearBanding in Jammed Systems, Eur. Phys. J. E 33, 183 (2010).

[27] J. Paredes, N. Shahidzadeh-Bonn, and D. Bonn, Shear Banding in Thixotropic and Normal Emulsions, J. Phys. Condens. Matter 23, 284116 (2011).

[28] P. Chaudhuri, L. Berthier, and L. Bocquet, Inhomogeneous Shear Flows in Soft Jammed Materials with Tunable Attractive Forces, Phys. Rev. E 85, 021503 (2012).

[29] T. Divoux, V. Grenard, and S. Manneville, Rheological Hysteresis in Soft Glassy Materials, Phys. Rev. Lett. 110, 018304 (2013).

[30] E. Irani, P. Chaudhuri, and C. Heussinger, Impact of Attractive Interactions on the Rheology of Dense Athermal Particles, Phys. Rev. Lett. 112, 188303 (2014).

[31] E. Irani, P. Chaudhuri, and C. Heussinger, Athermal Rheology of Weakly Attractive Soft Particles, Phys. Rev. E 94, 052608 (2016).

[32] R. Radhakrishnan, T. Divoux, S. Manneville, and S. M. Fielding, Understanding Rheological Hysteresis in Soft Glassy Materials, Soft Matter 13, 1834 (2017).

[33] A. D. S. Parmar, S. Kumar, and S. Sastry, Strain Localization Above the Yielding Point in Cyclically Deformed Glasses, Phys. Rev. X 9, 021018 (2019).

[34] T. Divoux, D. Tamarii, C. Barentin, and S. Manneville, Transient Shear Banding in a Simple Yield Stress Fluid, Phys. Rev. Lett. 104, 208301 (2010).

[35] R. L. Moorcroft and S. M. Fielding, Shear Banding in Time-Dependent Flows of Polymers and Wormlike Micelles, J. Rheol. 58, 103 (2014).

[36] R. Benzi, T. Divoux, C. Barentin, S. Manneville, M. Sbragaglia, and F. Toschi, Unified Theoretical and Experimental View on Transient Shear Banding, Phys. Rev. Lett. 123, 248001 (2019).

[37] C. Y. D. Lu, P. D. Olmsted, and R. C. Ball, Effects of Nonlocal Stress on the Determination of Shear Banding Flow, Phys. Rev. Lett. 84, 642 (2000).

[38] G. Picard, A. Ajdari, L. Bocquet, and F. Lequeux, Simple Model for Heterogeneous Flows of Yield Stress Fluids, Phys. Rev. E 66, 051501 (2002). 
[39] C. Masselon, A. Colin, and P. D. Olmsted, Influence of Boundary Conditions and Confinement on Nonlocal Effects in Flows of Wormlike Micellar Systems, Phys. Rev. E 81, 021502 (2010).

[40] K. Martens, L. Bocquet, and J.-L. Barrat, Spontaneous Formation of Permanent Shear Bands in a Mesoscopic Model of Flowing Disordered Matter, Soft Matter 8, 4197 (2012).

[41] S. Lerouge and P. D. Olmsted, Non-Local Effects in Shear Banding of Polymeric Flows, Front. Phys. 7, 246 (2020).

[42] M. Macaulay and P. Rognon, Viscosity of Cohesive Granular Flows, Soft Matter 17, 165 (2021).

[43] D. Fenistein and M. van Hecke, Wide Shear Zones in Granular Bulk Flow, Nature (London) 425, 256 (2003).

[44] A. Singh, V. Magnanimo, K. Saitoh, and S. Luding, Effect of Cohesion on Shear Banding in Quasistatic Granular Materials, Phys. Rev. E 90, 022202 (2014).

[45] M. Bouzid, M. Trulsson, P. Claudin, E. Clément, and B. Andreotti, Nonlocal Rheology of Granular Flows across Yield Conditions, Phys. Rev. Lett. 111, 238301 (2013).

[46] K. L. Johnson, K. Kendall, and A. D. Roberts, Surface Energy and the Contact of Elastic Solids, Proc. R. Soc. A 324, 301 (1971).

[47] A. Jarray, H. Shi, B. J. Scheper, M. Habibi, and S. Luding, Cohesion-Driven Mixing and Segregation of Dry Granular Media, Sci. Rep. 9, 13480 (2019).

[48] A. Gans, O. Pouliquen, and M. Nicolas, Cohesion-Controlled Granular Material, Phys. Rev. E 101, 032904 (2020).

[49] S. Mandal and D. V. Khakhar, A Study of the Rheology of Planar Granular Flow of Dumbbells Using Discrete Element Method Simulations, Phys. Fluids 28, 103301 (2016).

[50] S. Mandal and D. V. Khakhar, A Study of the Rheology and Micro-Structure of Dumbbells in Shear Geometries, Phys. Fluids 30, 013303 (2018).
[51] S. Mandal and D. V. Khakhar, Dense Granular Flow of Mixtures of Spheres and Dumbbells Down a Rough Inclined Plane: Segregation and Rheology, Phys. Fluids 31, 023304 (2019).

[52] O. Kuwano, R. Ando, and T. Hatano, Crossover from Negative to Positive Shear Rate Dependence in Granular Friction, Geophys. Res. Lett. 40, 1295 (2013).

[53] E. DeGiuli and M. Wyart, Friction Law and Hysteresis in Granular Materials, Proc. Natl. Acad. Sci. U.S.A. 114, 9284 (2017).

[54] I. S. Aranson and L.S. Tsimring, Continuum Theory of Partially Fluidized Granular Flows, Phys. Rev. E 65, 061303 (2002).

[55] K. Kamrin, Non-Locality in Granular Flow: Phenomenology and Modeling Approaches, Front. Phys. 7, 116 (2019).

[56] K. Kamrin and G. Koval, Nonlocal Constitutive Relation for Steady Granular Flow, Phys. Rev. Lett. 108, 178301 (2012).

[57] M. Bouzid, A. Izzet, M. Trulsson, E. Clément, P. Claudin, and B. Andreotti, Non-Local Rheology in Dense Granular Flows, Eur. Phys. J. E 38, 125 (2015).

[58] S. Li and D. L. Henann, Material Stability and Instability in Non-Local Continuum Models for Dense Granular Materials, J. Fluid Mech. 871, 799 (2019).

[59] J. Goyon, A. Colin, G. Ovarlez, A. Ajdari, and L. Bocquet, Spatial Cooperativity in Soft Glassy Flows, Nature (London) 454, 84 (2008).

[60] L. Bocquet, A. Colin, and A. Ajdari, Kinetic Theory of Plastic Flow in Soft Glassy Materials, Phys. Rev. Lett. 103, 036001 (2009).

[61] H. Perrin, C. Clavaud, M. Wyart, B. Metzger, and Y. Forterre, Interparticle Friction Leads to Nonmonotonic Flow Curves and Hysteresis in Viscous Suspensions, Phys. Rev. X 9, 031027 (2019). 